\title{
Blood Pressure Dynamics After Pubertal Suppression with Gonadotropin-Releasing Hormone Analogs Followed by Testosterone Treatment in Transgender Male Adolescents: A Pilot Study
}

\author{
Liat Perl, MD, Anat Segev-Becker, MD, ${ }^{1}$ Galit Israeli, MD, Erella Elkon-Tamir, MD, ${ }^{1,2}$ and Asaf Oren, MD ${ }^{1,2}$
}

\begin{abstract}
Purpose: We analyzed blood pressure (BP) changes in transgender male adolescents treated with gonadotropinreleasing hormone analogs (GnRHa) and after adding testosterone treatment.

Methods: This was a retrospective pilot study. Outcome measures included systolic BP (SBP) and diastolic BP (DBP) before and after GnRHa initiation and after adding testosterone.

Results: Fifteen transgender male adolescents received GnRHa. DBP percentiles increased significantly after GnRHa treatment (from 55.9\% \pm 26.4 to $73.6 \% \pm 9.4, p=0.019$ ). BP levels did not meet criteria for hypertension. DBP percentiles were restored after adding testosterone.

Conclusion: GnRHa may increase DBP in transgender male adolescents, and testosterone treatment may restore it. Further larger studies are indicated.
\end{abstract}

Keywords: blood pressure, gender dysphoria, gender-affirming hormone treatment, gonadotropin-releasing hormone analog, testosterone, transgender male adolescents

\section{Introduction}

$\mathbf{T}$ HE PROVISION OF TREATMENT of gender dysphoria (GD) in transgender youth has been expanding in recent years. The Endocrine Society Clinical Practice Guidelines and World Professional Association for Transgender Health (WPATH) Standards of Care endorse pubertal suppression with gonadotropin-releasing hormone analogs (GnRHa) from Tanner stage 2 of puberty in individuals experiencing GD with the onset of puberty. ${ }^{1,2}$ They also support initiating gender-affirming hormone treatment (testosterone or estradiol) at the age of legal consent $^{2}$ and even younger. ${ }^{1}$ GnRHa have been used in the treatment of central precocious puberty for several decades. Their benefits are well established, and their use is regarded as both safe and effective. ${ }^{3}$ Nevertheless, there is still concern about adverse effects on blood pressure (BP) and cardiac outcomes, body composition, bone health, and brain development in association with GnRHa treatment of youth with GD. ${ }^{1,4,5}$

The Endocrine Society Clinical Practice Guidelines recommend BP monitoring before and during treatment with GnRHa and/or treatment with testosterone. ${ }^{1}$ These recommendations are based on a few case reports of hypertension during GnRHa treatment in cisgender girls with precocious puberty. ${ }^{6-8}$ Data on BP in transgender youth treated with GnRHa are scarce. Three cases of GnRHa-induced arterial hypertension in transgender male adolescents were recently described in a single case series. ${ }^{9}$ Testosterone therapy was mostly reported to increase systolic $\mathrm{BP}(\mathrm{SBP})$ in transgender males, although the results were conflicting, ${ }^{10}$ with either increasing or unchanged effects on diastolic BP (DBP). ${ }^{11}$ The aim of this pilot study was to examine BP dynamics in a cohort of transgender male adolescents treated with GnRHa alone and after the addition of gender-affirming testosterone treatment.

\section{Patients and Methods}

This was a retrospective single-center observational study. The medical files of 48 transgender male adolescents who had sought medical attention due to GD at the Israeli Pediatric Gender Dysphoria Clinic, Dana-Dwek Children's Hospital, Tel Aviv Sourasky Medical Center between 2013 and 2018 were reviewed. The study was approved by the Tel

\footnotetext{
${ }^{1}$ Pediatric Endocrinology and Diabetes Unit, Dana-Dwek Children's Hospital, Tel Aviv Sourasky Medical Center, Tel Aviv, Israel.

${ }^{2}$ Sackler Faculty of Medicine, Tel Aviv University, Tel Aviv, Israel.

The study was presented as a poster presentation at the European Society for Paediatric Endocrinology, September 19-21, 2019, Vienna, Austria.
} 
Aviv Sourasky Medical Center Institutional Review Board, which waived informed consent for this retrospective data analysis.

Included in the analysis were all transgender male adolescents who were treated solely with GnRHa for $\geq 2$ months. $\mathrm{BP}$ assessments were recorded at the following time points: before pubertal suppression, after pubertal suppression, and, if applicable, after gender-affirming testosterone therapy. Of the total of 48 cases, 15 fulfilled these study inclusion criteria and 33 were excluded ( 21 had initiated GnRHa and testosterone therapy simultaneously, 8 did not initiate medical treatment, 3 had missing BP data, and 1 was on oral contraceptive therapy).

Medical treatment was administered in accordance with the Endocrine Society Guidelines ${ }^{1}$ and the WPATH Standards of Care. ${ }^{2}$ GnRHa treatment was offered to adolescents with GD who had reached at least Tanner stage 2 of breast development. Patients with GD who reached $\geq 14$ years of age were also offered gender-affirming hormone therapy. The pace of medical treatment was individually tailored according to the adolescent's requests, mental health personnel recommendations, and parental consent. All of the adolescents were offered medical nutrition counseling and psychosocial support as part of the routine standard of care. Clinic visits were scheduled to take place every 4 months.

Pubertal suppression therapy consisted of a depot preparation of the GnRHa D-Trp-6-LHRH (Decapeptyl; Ferring Pharmaceuticals Ltd., Malmö, Sweden) at a dose of $3.75 \mathrm{mg}$ administered by intramuscular injection every 4 weeks. Genderaffirming hormone therapy consisted of intramuscular testosterone enanthate $250 \mathrm{mg} / \mathrm{mL}$ (Testoviron ${ }^{\circledR}$ Depot; Bayer Israel Ltd.) at a starting dose of $50-100 \mathrm{mg}$ administered by intramuscular injection every 4 weeks.

Data extracted from medical records included vital signs, anthropometric measurements, and hormone levels (luteinizing hormone [LH], follicle-stimulating hormone [FSH], estradiol, and testosterone through levels). BP was measured during a clinic visit within 1-4 weeks of GnRHa and testosterone injections using a Welch Allyn Vital Signs Monitor VSM 300 (Welch Allyn, Inc., Beaverton, OR), and converted to BP percentiles according to assigned sex at birth (females) based on reference ranges in the Clinical Practice Guideline for Screening and Management of High Blood Pressure in Children and Adolescents. ${ }^{12}$ Normal BP was defined when $\mathrm{BP}$ values were $<90$ th percentile, prehypertension was defined by either SBP and/or DBP levels $\geq 90$ th percentile to $<95$ th percentile, and hypertension was defined by either SBP and/or diastolic BP levels $\geq 95$ th percentile. ${ }^{12}$

\section{Primary outcome measures}

Outcomes were changes in weight status (body mass index standard deviation score [BMI-SDS]) and BP levels (SBP and DBP percentiles) in study patients treated with GnRHa alone and after the addition of testosterone treatment.

\section{Statistical analyses}

Statistical analyses were performed using descriptive statistics, mean and SDs as standard central tendency, and dispersion determinations. Dynamics of outcome variables over time were analyzed with repeated measures analysis of variance or, when correcting for baseline covariate(s), with repeated measures analysis of covariance. A twosided $p$-value $<0.05$ was considered significant. SPSS v. 25.0 (IBM Corp., release date: August 8, 2017) was used to perform all analyses.

\section{Results}

The baseline anthropometric and hormone levels of the 15 transgender male adolescents included in the data analysis are presented in Table 1. Mean age at initiation of pubertal suppression was $14.4 \pm 1.0$ years. All 15 transgender males had clinical and laboratory evidence of Tanner stage $4 / 5$ puberty. Their weight status at the initiation of GnRHa treatment was normal (mean BMI-SDS 0.2 \pm 0.9 ), as were their BP levels and percentiles (mean SBP $115 \pm 7 \mathrm{~mm} \mathrm{Hg}$ [71 \pm 19 percentile] and mean DBP: $64 \pm 10 \mathrm{~mm} \mathrm{Hg}$ [56 \pm 26 percentile]).

The hypothalamic-pituitary-gonadal axis was successfully suppressed by GnRHa treatment in all 15 subjects. GnRHa was administered for a mean period of $3 \pm 1$ months. Weight status (BMI-SDS) did not increase significantly during GnRHa therapy $(p=0.198)$. DBP percentiles increased significantly after GnRHa treatment (from the $56.0 \pm 26.0$ percentile to $74.0 \pm 9.0$ percentile, $p=0.019$ ) (Fig. 1), and that increase remained significant after adjusting for the change in BMISDS ( $p=0.047)$. SBP percentiles did not change significantly, and the BP levels were within the normal range and did not meet the criteria for pediatric hypertension.

Testosterone treatment was initiated in 9 of the 15 transgender male adolescents at a mean age of $15.1 \pm 0.9$ years. After a mean treatment period of $4 \pm 2$ months, nadir testosterone levels increased significantly, reaching a mean of $7.1 \pm 4.6 \mathrm{nmol} / \mathrm{L}$. Weight status (BMI-SDS) did not change significantly. DBP percentiles decreased significantly after adding testosterone therapy (from $74.0 \pm 9.0$ to $56.0 \pm 17$ ) (Fig. 1), only after adjusting for the change in BMI-SDS $(p=0.033)$. DBP percentiles did not differ significantly compared with baseline, after testosterone treatment. The SBP percentiles did not change significantly. No significant correlations were found between BP percentiles and the testosterone, estradiol, LH, or FSH levels.

\section{Discussion}

Data on the effects of GnRHa on BP in transgender youth are very scarce. The results of this retrospective single-center observational pilot study revealed that pubertal suppression with GnRHa may increase DBP percentiles in transgender male adolescents, and that induction of puberty with testosterone treatment may restore DBP percentiles. Our current observations provide support for the Endocrine Society Clinical Practice Guidelines recommendation to monitor BP before and during treatment with GnRHa and testosterone. ${ }^{1}$

Our literature search yielded only three case reports of hypertension during GnRHa treatment that involved cisgender females with precocious puberty. Calcaterra et al. ${ }^{7}$ and Palma et al. ${ }^{6}$ described separately systolic and diastolic hypertension that developed in two girls treated with GnRHa for precocious puberty, and whose BP normalized after discontinuing treatment. The third case report described a previously normotensive 10 -year-old girl with clinically and genetically typical Williams-Beuren syndrome who presented with severe arterial hypertension 6 months after the initiation of GnRHa therapy for early normal puberty. ${ }^{8} \mathrm{~A}$ 
Table 1. Anthropometric Measurements and Hormone Concentrations of Patients at Baseline and After Treatment with Gonadotropin-Releasing Hormone Analogs and Testosterone

\begin{tabular}{|c|c|c|c|c|c|c|}
\hline \multirow[b]{2}{*}{ Parameter } & \multicolumn{3}{|c|}{ Pubertal suppression $(\mathrm{n}=15)$} & \multicolumn{3}{|c|}{ Gender-affirming hormone therapy $(\mathrm{n}=9)$} \\
\hline & $\begin{array}{c}\text { Before GnRHa } \\
\text { treatment }\end{array}$ & $\begin{array}{l}\text { After GnRHa } \\
\text { treatment }\end{array}$ & $\mathrm{p}$ & $\begin{array}{l}\text { Before addition of } \\
\text { testosterone treatment }\end{array}$ & $\begin{array}{c}\text { After addition of } \\
\text { testosterone treatment }\end{array}$ & $\mathrm{p}$ \\
\hline Age (years) & $14.4 \pm 1.0$ & $14.8 \pm 1.0$ & & $15.1 \pm 0.9$ & $15.8 \pm 0.9$ & \\
\hline BMI $\left(\mathrm{kg} / \mathrm{m}^{2}\right)$ & $21.3 \pm 4.7$ & $22.0 \pm 4.8$ & 0.024 & $23.3 \pm 5.6$ & $24.2 \pm 4.6$ & 0.199 \\
\hline BMI-SDS & $0.2 \pm 0.9$ & $0.4 \pm 0.9$ & 0.198 & $0.6 \pm 1.0$ & $0.8 \pm 0.8$ & 0.207 \\
\hline SBP $(\mathrm{mmHg})$ & $115 \pm 7$ & $116 \pm 5$ & 0.532 & $117 \pm 4$ & $116 \pm 6$ & 0.776 \\
\hline SBP percentiles & $71 \pm 19$ & $76 \pm 14$ & 0.443 & $76 \pm 14$ & $72 \pm 21$ & 0.57 \\
\hline $\mathrm{DBP}(\mathrm{mmHg})$ & $64 \pm 10$ & $71 \pm 3.0$ & 0.009 & $71 \pm 3.0$ & $68 \pm 5$ & 0.212 \\
\hline DBP percentiles & $56 \pm 26$ & $74 \pm 9.0$ & 0.019 & $74 \pm 9.0$ & $56 \pm 17$ & 0.059 \\
\hline $\mathrm{LH}(\mathrm{mIU} / \mathrm{mL})$ & $6.6 \pm 6.0$ & $0.2 \pm 0.4$ & 0.02 & $0.2 \pm 0.3$ & $0.1 \pm 0.2$ & 0.429 \\
\hline FSH (mIU/mL) & $6.2 \pm 2.4$ & $2.5 \pm 1.2$ & $<0.001$ & $2.4 \pm 1.0$ & $2.0 \pm 1.3$ & 0.341 \\
\hline Estradiol (pmol/L) & $264.6 \pm 238.2$ & $0 \pm 0$ & 0.003 & $0.0 \pm 0.0$ & $38.6 \pm 42.0$ & 0.035 \\
\hline Testosterone (nmol/L) & $1.4 \pm 1.2$ & $0.4 \pm 0.4$ & 0.03 & $0.4 \pm 0.5$ & $7.1 \pm 4.6$ & 0.007 \\
\hline
\end{tabular}

Data shown are mean \pm SD. For estradiol, undetectable levels are entered as 0 .

BMI, body mass index; BMI-SDS, BMI standard deviation score; DBP, diastolic blood pressure; FSH, follicle-stimulating hormone; GnRHa, gonadotropin-releasing hormone analogs; LH, luteinizing hormone; SBP, systolic blood pressure; SD, standard deviation.

few small studies on adult females treated with GnRHa for symptomatic fibroids or endometriosis or before in vitro fertilization did not show a significant increase in BP. ${ }^{13,14}$

Klink et al. described a single case series of hypertension in three transgender male adolescents after initiation of treatment with GnRHa: (1) an 11.8-year-old who developed systolic and diastolic hypertension, confirmed after 16 months of GnRHa treatment. A diagnostic workup showed papilledema and elevated intracranial pressure with a normal magnetic resonance imaging scan. (2) An 18-year-old transgender male who developed systolic and diastolic hypertension after 3 months of
GnRHa treatment. (3) A 12.5-year-old who developed systolic hypertension after 3 months of GnRHa therapy. ${ }^{9}$

A plausible mechanism in which GnRHa might cause hypertension in assigned-at-birth females is hypoestrogenism and hypoprogesteronism. It has been established that estrogen influences the vascular system by inducing vasodilatation, increasing nitric oxide bioavailability, and inhibiting vascular remodeling processes and vascular response to injury. Moreover, estrogen modulates the renin-angiotensin-aldosterone system and the sympathetic system. These mechanisms have a protective effect on atrial stiffness. ${ }^{15}$ Furthermore, progesterone has been shown
FIG. 1. Diastolic blood pressure percentile trends during GnRHa and testosterone treatment. Individual diastolic blood pressure before pubertal suppression with GnRHa, after pubertal suppression but before genderaffirming hormone therapy, and after gender-affirming hormone therapy in 15 transgender male adolescents. Testosterone treatment was initiated in nine individuals. $* p=0.019, p=0.047$ after BMI-SDS change adjustment. $* * p=0.03$ after BMISDS change adjustment. BMI-SDS, body mass index standard deviation score; GnRHa, gonadotropinreleasing hormone analogs.

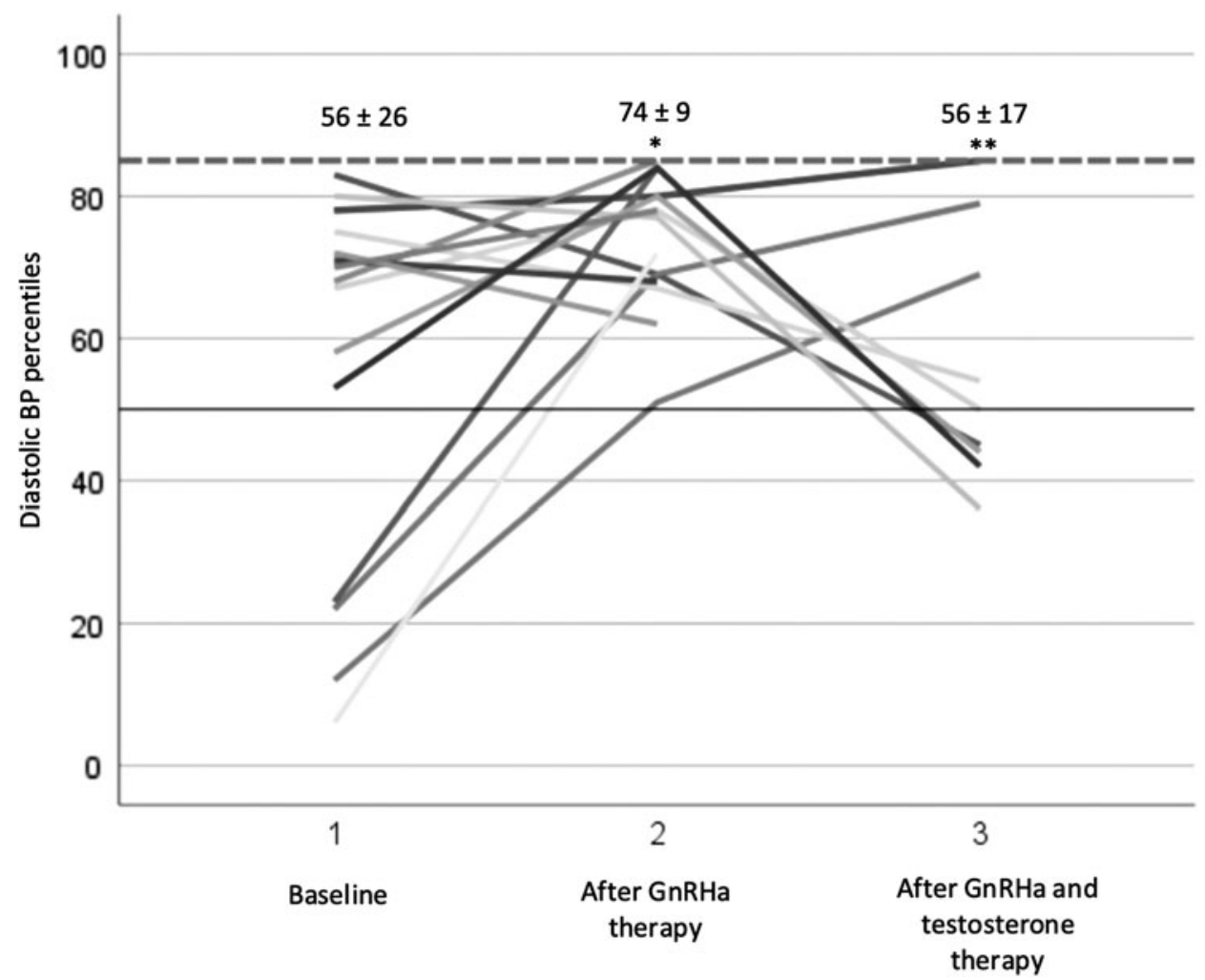


to cause endothelium-dependent vascular dilation. ${ }^{15}$ As treatment with GnRHa at Tanner stage 4-5 in transgender males causes abrupt hypoestrogenism and hypoprogesteronism, it might be anticipated that vascular dilatation and stiffness would be affected and that BP would increase as a result. As DBP reflects the peripheral resistance that the heart must overcome to eject blood, ${ }^{16}$ it is probable that low estrogen and progesterone levels primarily affect DBP. This hypothesis supports our current findings of DBP dynamics with preserved SBP levels. Although DBP percentiles increased significantly in our study, it is noteworthy that they did not meet the criteria for pediatric hypertension. This finding is encouraging, but nevertheless mandates monitoring BP during treatment.

Our sample was normotensive at baseline and had normal weight status. There was a mild but nonsignificant weight status change after GnRHa and testosterone treatment, unlike other studies that have demonstrated an increase in BMI after such treatment. ${ }^{10,17,18}$ It would be informative to assess BP in a sample of hypertensive and/or overweight transgender youth at baseline to determine whether specific subgroups are at an increased risk.

Our study results demonstrated that induction of puberty with testosterone treatment restored and lowered DBP percentiles compared with DBP percentiles after treatment with GnRHa. We speculate that this effect could be due to local aromatase activity in vascular tissue. Aromatase has been shown to be present in vascular smooth muscle cells of the human aorta and pulmonary arteries, as well as in smooth muscle and endothelial cells of the vena cava, ${ }^{19}$ possibly converting exogenous testosterone to estrogens locally, and restoring estrogen's protective properties on BP. Our comparison between the SBP and DBP percentiles at baseline and after adding testosterone treatment to GnRHa revealed no significant treatment-related change. Other studies that have assessed the impact of various testosterone formulations on BP levels in transgender men demonstrated mixed results. ${ }^{10,11,20}$ In transgender male adolescents, studies have shown unchanged SBP and DBP after testosterone treatment in accordance with our study. ${ }^{18,21}$

\section{Limitations}

Our study has several limitations. It was retrospective in design, and it included a small number of patients, and an even smaller number of patients who received testosterone treatment. Furthermore, the duration of follow-up on GnRHa treatment and after the addition of testosterone was quite short, which precluded our ability to discuss long-term effects of GnRHa and testosterone on BP and to assess whether the effects that were observed were transient. Despite the short duration of follow-up on GnRHa treatment, however, gonadotropins were fully suppressed. The maximal effect of testosterone on BP could not be determined because the BP assessment was done after a relatively short period of testosterone treatment during which testosterone dose and blood levels had not yet reached adult ranges. In addition, the BP measurements were taken at the start of each clinic visit, and might have been affected by factors, such as anticipation and stress, associated with the visit. Unfortunately, 24-hour ambulatory BP monitoring to determine circadian rhythm and variability had not been performed.

\section{Conclusions}

Based on our findings in this pilot study, we suggest that pubertal suppression with GnRHa may increase DBP in transgender male adolescents, but does not cause hypertension, and that the induction of puberty with gender-affirming testosterone treatment may restore DBP percentiles. Further studies with larger cohorts and more rigorous methods of BP assessment are needed to elucidate the effect of BP dynamics on the metabolic and cardiovascular consequences for adolescents undergoing hormone treatment for GD.

\section{Acknowledgments}

We gratefully thank Dr. Yael Lebenthal for reviewing the article and providing helpful comments and Esther Eshkol for editing the article.

\section{Author Disclosure Statement}

No competing financial interests exist.

\section{Funding Information}

No funding was received for this article.

\section{References}

1. Hembree WC, Cohen-Kettenis PT, Gooren L, et al.: Endocrine treatment of gender-dysphoric/gender-incongruent persons: An Endocrine Society Clinical Practice Guideline. J Clin Endocrinol Metab 2017;102:3869-3903.

2. Coleman E, Bockting W, Botzer M, et al.: Standards of care for the health of transsexual, transgender, and gendernonconforming people, Version 7. Int J Transgend 2012;13: 165-232.

3. Carel JC, Eugster EA, Rogol A, et al.: Consensus statement on the use of gonadotropin-releasing hormone analogs in children. Pediatrics 2009;123:752-762.

4. T'Sjoen G, Arcelus J, Gooren L, et al.: Endocrinology of transgender medicine. Endocr Rev 2019;40:97-117.

5. Chew D, Anderson J, Williams K, et al.: Hormonal treatment in young people with gender dysphoria: A systematic review. Pediatrics 2018;141:pii: e20173742.

6. Palma L, Gaudino R, Cavarzere P, Antoniazzi F: Does the risk of arterial hypertension increase in the course of triptorelin treatment? J Pediatr Endocrinol Metab 2020;33:449-452.

7. Calcaterra V, Mannarino S, Corana G, et al.: Hypertension during therapy with triptorelin in a girl with precocious puberty. Indian J Pediatr 2013;80:884-885.

8. Siomou E, Kosmeri C, Pavlou M, et al.: Arterial hypertension during treatment with triptorelin in a child with WilliamsBeuren syndrome. Pediatr Nephrol 2014;29:1633-1636.

9. Klink D, Bokenkamp A, Dekker C, Rotteveel J: Arterial hypertension as a complication of triptorelin treatment in adolescents with gender dysphoria. Endocrinol Metab Int J 2015;2:36-38.

10. Velho I, Fighera TM, Ziegelmann PK, Spritzer PM: Effects of testosterone therapy on BMI, blood pressure, and laboratory profile of transgender men: A systematic review. Andrology 2017;5:881-888.

11. Irwig MS: Cardiovascular health in transgender people. Rev Endocr Metab Disord 2018;19:243-251.

12. Flynn JT, Kaelber DC, Baker-Smith CM, et al.: Clinical practice guideline for screening and management of high blood pressure in children and adolescents. Pediatrics 2017;140:pii: e20171904. 
13. Eckstein N, Pines A, Fisman EZ, et al.: The effect of the hypoestrogenic state, induced by gonadotropin-releasing hormone agonist, on doppler-derived parameters of aortic flow. J Clin Endocrinol Metab 1993;77:910-912.

14. Ferreira RA, Vieira CS, Rosa-E-Silva JC, et al.: Effects of the levonorgestrel-releasing intrauterine system on cardiovascular risk markers in patients with endometriosis: A comparative study with the $\mathrm{GnRH}$ analogue. Contraception 2010;81:117-122.

15. Di Giosia P, Giorgini P, Stamerra CA, et al.: Gender differences in epidemiology, pathophysiology, and treatment of hypertension. Curr Atheroscler Rep 2018;20:13.

16. Li Y, Wei FF, Wang S, et al.: Cardiovascular risks associated with diastolic blood pressure and isolated diastolic hypertension. Curr Hypertens Rep 2014;16:489.

17. Schagen SE, Cohen-Kettenis PT, Delemarre-van de Waal HA, Hannema SE: Efficacy and safety of gonadotropin-releasing hormone agonist treatment to suppress puberty in gender dysphoric adolescents. J Sex Med 2016;13:1125-1132.

18. Jarin J, Pine-Twaddell E, Trotman G, et al.: Cross-sex hormones and metabolic parameters in adolescents with gender dysphoria. Pediatrics 2017;139:pii: e20163173.
19. Simpson ER, Clyne C, Rubin G, et al.: Aromatase-A brief overview. Annu Rev Physiol 2002;64:93-127.

20. Vita R, Settineri S, Liotta M, et al.: Changes in hormonal and metabolic parameters in transgender subjects on cross-sex hormone therapy: A cohort study. Maturitas 2018; 107:92-96.

21. Olson-Kennedy J, Okonta V, Clark LF, Belzer M: Physiologic response to gender-affirming hormones among transgender youth. J Adolesc Health 2018;62:397-401.

Address correspondence to:

Liat Perl, MD

Pediatric Endocrinology and Diabetes Unit

Dana-Dwek Children's Hospital

Tel Aviv Sourasky Medical Center

6 Weizmann Street

Tel Aviv 64239-06

Israel

E-mail: liatpe@tlvmc.gov.il 